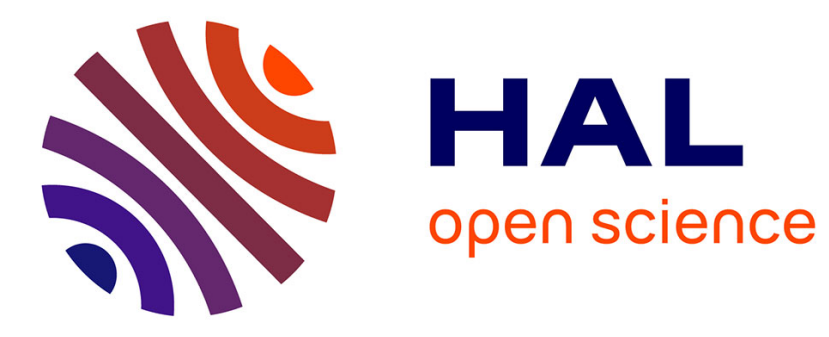

\title{
A moving magnetic mirror to slow down a bunch of atoms
}

Gaël Reinaudi, Zhaoying Wang, Antoine Couvert, Thierry Lahaye, David Guéry-Odelin

\section{- To cite this version:}

Gaël Reinaudi, Zhaoying Wang, Antoine Couvert, Thierry Lahaye, David Guéry-Odelin. A moving magnetic mirror to slow down a bunch of atoms. The European Physical Journal D: Atomic, molecular, optical and plasma physics, 2006, 40, pp.405. 10.1140/epjd/e2006-00244-6 . hal-00090483v2

\section{HAL Id: hal-00090483 \\ https://hal.science/hal-00090483v2}

Submitted on 15 Sep 2008

HAL is a multi-disciplinary open access archive for the deposit and dissemination of scientific research documents, whether they are published or not. The documents may come from teaching and research institutions in France or abroad, or from public or private research centers.
L'archive ouverte pluridisciplinaire HAL, est destinée au dépôt et à la diffusion de documents scientifiques de niveau recherche, publiés ou non, émanant des établissements d'enseignement et de recherche français ou étrangers, des laboratoires publics ou privés. 


\title{
A moving magnetic mirror to slow down a bunch of atoms
}

\author{
G. Reinaudi, Z. Wang, A. Couvert, T. Lahaye and D. Guéry-Odelin \\ Laboratoire Kastler Brossel $^{\mathrm{a}}$, Département de Physique de l'Ecole Normale Supérieure, \\ 24 rue Lhomond, 75005 Paris, France
}

September 15, 2008

\begin{abstract}
A fast packet of cold atoms is coupled into a magnetic guide and subsequently slowed down by reflection on a magnetic potential barrier ('mirror') moving along the guide. A detailed characterization of the resulting decelerated packet is performed. We show also how this technique can be used to generate a continuous and intense flux of slow, magnetically guided atoms.
\end{abstract}

PACS. $\quad 32.80 . \mathrm{Pj}, 42.50 . \mathrm{Vk}, 03.75 . \mathrm{Be}$

\section{Introduction}

Ultracold and slow beams have a huge potential in metrology, matter wave interferometry and nanolithography [1]. The achievement of an ultracold beam of neutrons was made possible by specularly reflecting neutrons from a Ni-surface which moved along the beam direction [2]. This breakthrough was followed by many achievements, among which neutron optics experiments [3] and neutron interferometry experiments [4].

In another context, the deceleration of a pulsed supersonic helium beam through reflection on crystalline atomic mirrors mounted on a high speed spinning rotor is currently being investigated [5, with also the goal of reaching a very intense source for atom optics experiments.

In this paper, we report on the slowing down of packets of atoms injected into a magnetic guide through their specular reflection from a moving magnetic mirror, and we also demonstrate the use of this technique to generate a high flux of slow and cold magnetically guided atoms. Such a beam is of crucial importance in order to realize a $\mathrm{cw}$ atom laser by implementing the forced evaporative cooling technique on a magnetically guided beam, as proposed in Ref. [6] and experimentally investigated in [7].

In the reference frame of the mirror, the atomic velocity simply changes sign after the reflection, going from $v_{\mathrm{i}}-v_{\mathrm{m}}$ to $v_{\mathrm{m}}-v_{\mathrm{i}}$, where $v_{\mathrm{i}}$ is the relatively high mean initial velocity of the packet and $v_{\mathrm{m}}$ is the mirror velocity. In the laboratory frame, the final velocity of the packet is thus:

$$
v_{\mathrm{f}}=2 v_{\mathrm{m}}-v_{\mathrm{i}} .
$$

Magnetic mirrors are based on the Zeeman interaction between an inhomogeneous magnetic field and the

\footnotetext{
${ }^{a}$ Unité de Recherche de l'Ecole Normale Supérieure et de l'Université Pierre et Marie Curie, associée au CNRS.
}

atomic magnetic dipole moment. Atoms in the low-fieldseeking state are then reflected elastically from high magnetic field regions. Magnetic mirrors for atom optics have been implemented in various ways, using floppy disks [8], videotapes [9], permanent magnets arrays [10] or microelectromagnets [11.

Moving mirrors for cold atoms have been studied up to now using a time-modulated, blue-detuned evanescent light wave propagating along the surface of a glass prism. This technique has been used to demonstrate atom optics manipulation: focussing of atomic trajectories, formation of multiple images of a point source, and phasemodulation of de Broglie waves $[12$.

This paper is organized as follows. In section 2 we describe the experimental setup. In section 3 we present our data on the slowing down of a single atomic packet through its collision with the moving mirror. In section 4 we detail the model that we have developed to analyse our experimental data. Finally, we discuss our preliminary results on the generation of a very slow and intense atomic beam using this technique.

\section{Experimental setup}

The experimental setup is schematically illustrated in Fig. 1 a (see also [7]).

Atomic packets of a few $10^{9}$ cold ${ }^{87} \mathrm{Rb}$ atoms are prepared in a magneto-optical trap fed by a Zeeman slower. The cloud is then set in motion at an adjustable velocity $v_{\mathrm{i}}$ by the moving molasses technique, and optically pumped into the weak-field-seeking ground state $\mid F=$ $\left.1, m_{F}=-1\right\rangle$ with magnetic moment $\mu=\mu_{B} / 2$ where $\mu_{B}$ is the Bohr magneton. It is launched towards the entrance of a 4.5 meters long magnetic guide. This guide, placed inside an ultra high vacuum chamber, consists of four parallel water-cooled copper tubes (see Figs. Ila and 
(a)

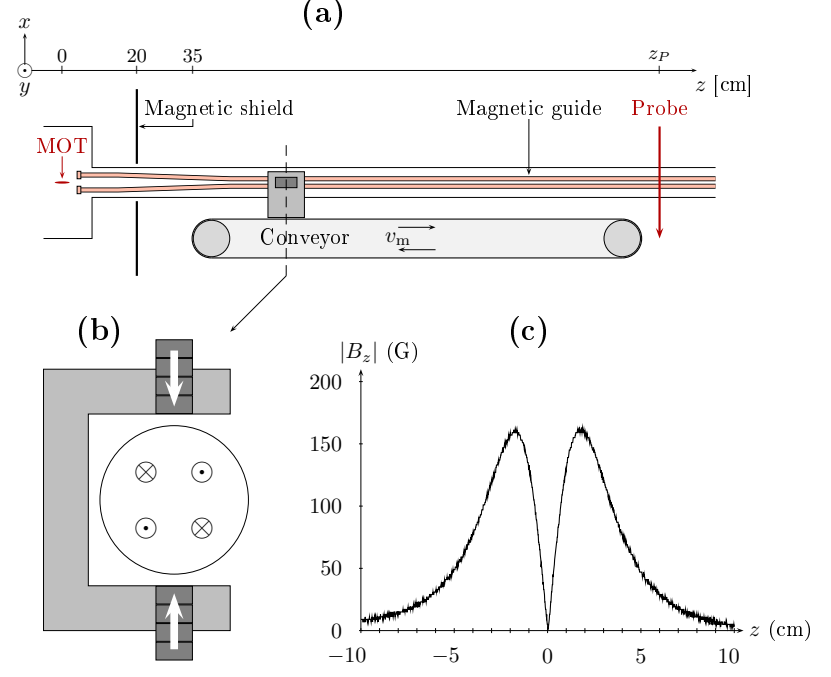

Fig. 1. (a) Overview of the experimental setup. (b) Transverse cut that illustrates the 4 copper tubes of the magnetic guide in the UHV chamber, in presence of the magnetic mirror generated by a pair of magnets facing each other with opposite magnetization. (c) Modulus of the longitudinal (along the $z$ axis) field generated by the magnets.

17b). For the results given below, a current $I=200 \mathrm{~A}$ per tube was used, generating a transverse magnetic field $B_{\mathrm{g}}=(b x,-b y, 0)$ with a gradient of $b=500 \mathrm{G} / \mathrm{cm}$. At the beginning of the magnetic guide, the packet is slowed down by undergoing a collision with a moving magnetic mirror.

This mirror is made of a pair of rare-earth (Nd-Fe-B) permanent magnets placed on a U-shaped support (see Fig. 1 1 b). The field magnitude controls the reflection of atoms in the adiabatic regime when the magnetic moment follows the local field direction. The chosen configuration with magnets facing each other with an opposite magnetization provides a longitudinal potential hill of height $\mu B_{\max }$, with $B_{\max } \simeq 160 \mathrm{G}$. This symmetric configuration minimizes the transverse magnetic fields, thus avoiding a transverse deflection of the trajectories during the reflection. We have plotted on Fig. 11c the measured absolute value of the longitudinal magnetic field component of the mirror.

The transverse potential experienced by the atoms in the magnetic guide is linear far away from the mirror as a result of the two-dimensional quadrupolar configuration chosen to confine transversally the atoms. The contribution of the mirror to the longitudinal field tends mainly to smooth out the bottom of the potential, resulting in a transverse harmonic confinement, while essentially not modifying the transverse gradient provided by the magnetic guide (the correction is less than $5 \%$ for our range of parameters). The magnetic mirror is fixed on a 1.2 meter long conveyor belt parallel to the guide axis, which allows to control its velocity $v_{\mathrm{m}}$ in a range of $20 \mathrm{~cm} / \mathrm{s}$ to $120 \mathrm{~cm} / \mathrm{s}$. The day-to-day velocity fluctuations are less than $2 \%$. In order to control the position of the collision between the packet and the moving magnetic mirror, we (a)

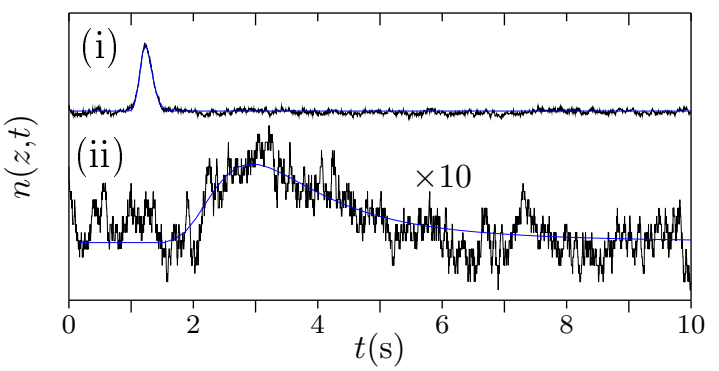

(b)

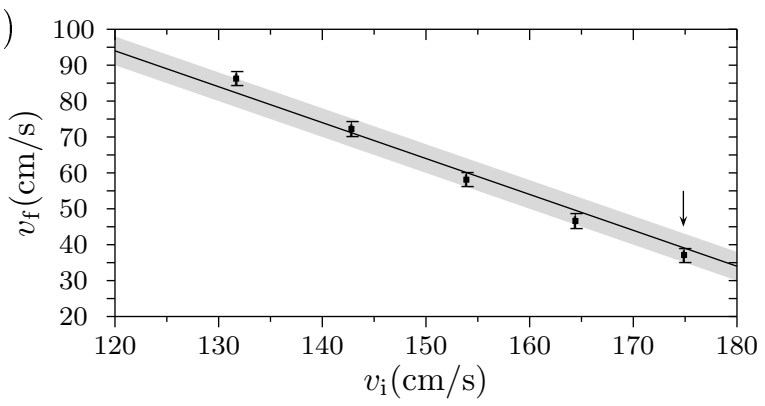

Fig. 2. (a) (i) Absorption signal from the probe placed at 1.75 meter from the MOT location. The time origin corresponds to the launching of the packet. The injection velocity of the packet is $142 \mathrm{~cm} / \mathrm{s}$. (ii) Absorption signal for a packet prepared in the same conditions, but that has undergone a collision with the magnetic mirror moving at a velocity $v_{\mathrm{m}}=86 \pm 2$ $\mathrm{cm} / \mathrm{s}$. (b) Measured final velocity of the packet as a function of the injection velocity for a mirror velocity of $107 \pm 2 \mathrm{~cm} / \mathrm{s}$. The straight line corresponds to the prediction of equation (11), the grey area accounts for the mirror velocity uncertainty. The arrow in (b) corresponds to parameters for which $95 \%$ of the initial kinetic energy of the packet has been removed through the reflection on the mirror.

synchronize the launching of the cloud into the guide with respect to the mirror motion.

The MOT region is protected by a magnetic shield from the influence of the magnetic mirror. Atoms enter the conveyor area after a propagation of $\sim 20 \mathrm{~cm}$ into the guide. We measure the characteristic of a given packet of atoms by monitoring the absorption of a probe beam positioned at $z_{P}=1.75 \mathrm{~m}$ (unless otherwise stated) from the MOT location (see Fig. 1a). To detect the atoms, we scan, 70 times per second, the frequency of the probe across the $\left|5^{2} \mathrm{~S}_{1 / 2}, F=1\right\rangle \rightarrow\left|5^{2} \mathrm{P}_{3 / 2}, F^{\prime}=0,1,2\right\rangle$ open transitions, at a rate of about $600 \mathrm{MHz} / \mathrm{ms}$. We monitor the maximum of this absorption spectrum with a sample-and-hold electronic circuit, which generates a data point for the absorption every $15 \mathrm{~ms}$. This technique reveals to be remarkably insensitive to the inhomogeneity of the magnetic field in the probe region, leading to a reliable and robust signal proportional to the local atomic density [7].

\section{Experimental results}

When the relative velocity of the atoms with respect to the mirror is larger than $\sim\left(2 \mu B_{\max } / m\right)^{1 / 2}$, atoms pass over the potential hill. We have indeed observed this ef- 
fect by launching a packet of atoms at this critical velocity on a motionless mirror. Due to the dispersion of longitudinal velocities in the packet, the packet was split into reflected and transmitted ones, in good agreement with the measured height of the magnetic potential barrier.

The absorption time-of-flight signal shown in Fig. 22a (i) corresponds to a packet injected into the magnetic guide at a velocity $v_{\mathrm{i}}=142 \mathrm{~cm} / \mathrm{s}$ with an initial longitudinal velocity dispersion of $\Delta v=12 \mathrm{~cm} / \mathrm{s}$ with no mirror present. The signal obtained after the collision with the mirror moving at a velocity $v_{\mathrm{m}}=86 \pm 2 \mathrm{~cm} / \mathrm{s}$ is depicted on Fig. 2 a (ii). The packet of atoms has clearly been slowed down. Indeed the measured final mean velocity is $\sim 35 \mathrm{~cm} / \mathrm{s}$, in good agreement with (11). For this specific example, the maximum of the absorption peak is delayed by approximately two seconds. The packet had consequently more time to spread out which justifies the increase of its width and the decrease of its height.

We have performed this experiment for different values of the initial velocities $v_{\mathrm{i}}$ and for two values of the mirror velocity $\left(v_{\mathrm{m}}=86 \pm 2 \mathrm{~cm} / \mathrm{s}\right.$ and $\left.107 \pm 2 \mathrm{~cm} / \mathrm{s}\right)$. The range of velocities that we have investigated was essentially dictated by two constraints: (i) The finite barrier height $\mu B_{\max }$, which limits the range of relative velocities between the packet and the mirror that can be studied, (ii) The length over which the collision occurs, which is limited by the size of the conveyor. In addition, the finite lifetime of the atoms due to collisions with the residual gas, combined with the sensitivity of the detection, limits ultimately the range of parameters over which reliable data can be obtained.

The measured final velocity $v_{\mathrm{f}}$ of the packet after its collision with the moving mirror is shown on Fig. 20 b as a function of its injection velocity $v_{\mathrm{i}}$ for a mirror velocity $107 \pm 2 \mathrm{~cm} / \mathrm{s}$. We have been able to slow packets with an initial velocity of $175 \mathrm{~cm} / \mathrm{s}$ down to $35 \mathrm{~cm} / \mathrm{s}$. Through the collision, we thus remove up to $95 \%$ of the longitudinal kinetic energy (experimental point indicated by a vertical arrow).

\section{Data analysis}

The solid and smooth line superimposed on the experimental data (see Fig. 2a) results from a simple analytical model that provides a good quantitative understanding of the collision between the packet and the moving magnetic mirror, as well as a flexible tool to analyze our experimental data.

In this model, we assume that the spatial distribution of the atoms is initially a Dirac function. This assumption is reasonable in the range of parameters experimentally investigated, since the size of the packet during the collision is dictated essentially by the initial velocity dispersion.

The initial joint distribution in position and velocity of the model is thus taken in the form: $\pi_{0}\left(z, v_{z}\right)=$ $N \delta(z) p_{0}\left(v_{z}\right)$ where $p_{0}\left(v_{z}\right)$ is the velocity distribution. In practice we use a gaussian function normalized to unity with a center velocity $v_{\mathrm{i}}$, and a velocity dispersion $\Delta v$. The magnetic mirror is modeled by an infinite repulsive potential wall. If the mirror is initially positioned at $z=d$, a particle initially positioned at $z_{i}<d$ with initial velocity $v_{z}$ is reflected after a free propagation over a distance $z^{*}=$ $v_{z}\left(d-z_{i}\right) /\left(v_{z}-v_{\mathrm{m}}\right)$, at time $t^{*}=\left(d-z_{i}\right) /\left(v_{z}-v_{\mathrm{m}}\right)$, and its velocity after the collision is, from (1), $v_{z}^{\prime}=2 v_{\mathrm{m}}-v_{z}$. In Fig. 3a, we provide an example of the packet evolution through a plot in the phase-space $\left(z, v_{z}\right)$. We have plotted the results of a Monte Carlo numerical simulation with $d=0.3 \mathrm{~m}$ at times $t=0$ and at $t=0.884 \mathrm{~s}$ where $93 \%$ of the atoms have already been reflected. In this example, the packet (i) has an initial velocity of $\left\langle v_{z}\right\rangle=142 \mathrm{~cm} / \mathrm{s}$, a velocity dispersion $\Delta v_{z}=12 \mathrm{~cm} / \mathrm{s}$, and a dispersion in position $\Delta r=0.02 \mathrm{~m}$. Those parameters are the ones of the experimental data of Fig. 2a. The density profile at time $t=0.884 \mathrm{~s}$ given by the numerical simulation performed with the real initial size, is in excellent agreement with our simple analytical model predictions without any adjustable parameter as shown on Fig. $3 \mathrm{~b}$.

Position-velocity correlations build up during the propagation: particles with a larger initial velocity are reflected earlier, which results in the inclined elliptical shape of the cloud in the phase-space plot. The reflection on the mirror acts to some extent as a translation in phase space while keeping the volume constant as expected from Liouville's theorem [13. In Fig. 3a atoms of class (ii) belong to the low velocity tail of the initial distribution and have not yet been reflected, while atoms of class (iii) have been significantly slowed down through their collision with the moving mirror.

The density profile as a function of position and time is derived from our model:

$$
\begin{aligned}
n(z, t)= & N \frac{\Theta\left(v_{\mathrm{m}} t+d-z\right)}{t}\left[p_{0}\left(\frac{z}{t}\right)\right. \\
& \left.+p_{0}\left(2 v_{\mathrm{m}}+\frac{2 d-z}{t}\right)\right]
\end{aligned}
$$

The Heaviside step function $\Theta$ in the prefactor means that all particles are always located before the mirror. The first term is the contribution of non reflected atoms, while the second accounts for the reflected particles.

This formula has been used to analyze our experimental data with a gaussian form for $p_{0}$. The agreement is very good with all our set of data. The value of the initial velocity and of the velocity dispersion are deduced from the propagation of the packet with no mirror present. We then plug the obtained values into our model to deduce the characteristics of the slowed packet after its interaction with the moving mirror (see Fig. 2a). In practice, we measure the instantaneous velocity of the conveyor belt (i.e. of the mirror), and the adjustable parameters for the fit are the final velocity, the amplitude of the absorption signal and an effective initial position of the mirror which accounts for the smoothness of the real mirror.

The evolution with time of the velocity distribution is obtained by separating the contribution from atoms that have not yet undergone a reflection, with the ones that have. At a given time $t$ after the launch of the packet, the atoms with a velocity larger than $\tilde{v}=v_{\mathrm{m}}+d / t$ have 

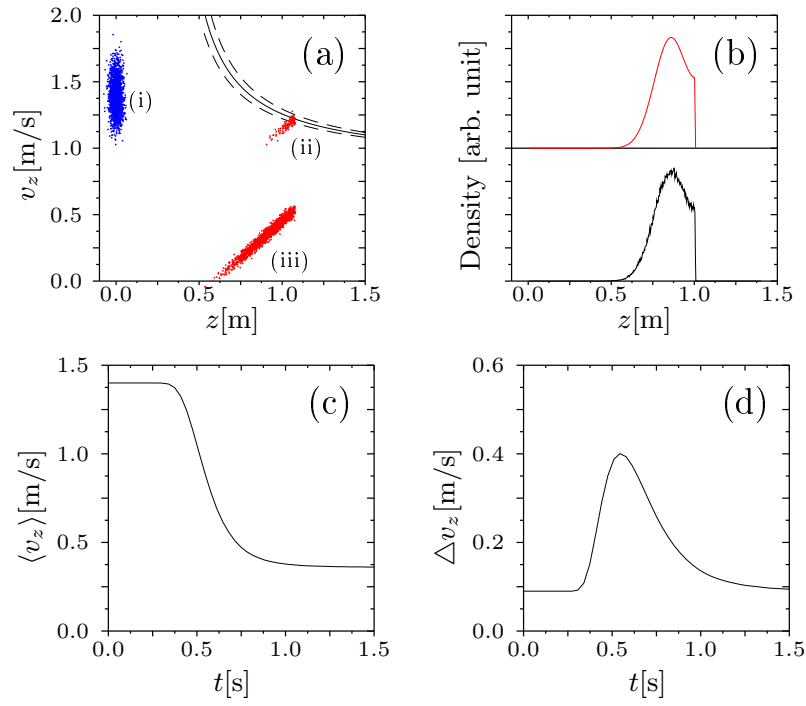

Fig. 3. Collision of a packet having an initial velocity $v_{\mathrm{i}}=142$ $\mathrm{cm} / \mathrm{s}$ and a velocity dispersion $\Delta v_{z}=12 \mathrm{~cm} / \mathrm{s}$ with an infinite repulsive wall moving at a velocity $v_{m}=88 \mathrm{~cm} / \mathrm{s}$. Initially the mirror is at a distance of $d=0.3 \mathrm{~m}$ from the initial mean position of the packet $(z=0)$. (a) Atomic distribution in the phase-space (i) at time $t=0$, and at time $t=0.884 \mathrm{~s}$ for (ii) and (iii). Atoms that belong to the class (ii) have not yet been reflected, while the atoms from the class (iii) have been slowed down through their collision with the moving mirror. The curve in solid line represents the position where the collision occurs for a given initial velocity assuming that the initial position of the atom is $z=0$. The zone delimited by the dashed lines accounts for the initial dispersion of the size of the packet. (b) Atomic density profile of the packet after a propagation time $t=0.884 \mathrm{~s}$. Upper graph: our model. Lower graph: Monte Carlo simulation. (c) Mean velocity of the packet as a function of time. (d) Velocity dispersion as a function of time.

been reflected. We deduce from our model the expression for the instantaneous velocity distribution $p\left(v_{z}, t\right)$ : $p\left(v_{z}, t\right)=p_{0}\left(v_{z}\right) \Theta\left(\tilde{v}-v_{z}\right)+p_{0}\left(2 v_{\mathrm{m}}-v_{z}\right) \Theta\left(2 v_{\mathrm{m}}-\tilde{v}-v_{z}\right)$. Starting with this relation, one readily obtains the expression of the mean velocity $\left\langle v_{z}\right\rangle(t)$, and of the dispersion $\Delta v_{z}(t)$. We plot on Figs. 3ic and 3d those quantities for the experimental parameters of Fig. 2a. The mean velocity decrease occurs on a relatively short time scale. The velocity dispersion turns out to not be a good quantity to characterize the packet during the collision. Indeed, the coexistence of slowed and fast particles results in a transient artificial increase of the dispersion. However, an important feature is that the velocity dispersion recovers its initial value after the collision with the moving mirror.

This technique is intrinsically pulsed and this is the reason why it has to be contrasted with other methods that can be used to slow down a beam of atoms using an upward slope or/and a tapered section with an increasing strength of the transverse confinement over a portion of the guide. In the latter techniques, the longitudinal velocity dispersion increases, while the mean velocity decreases 14 since the Liouville theorem applied to a continuous beam dictates in one-dimension the conservation of the product $\bar{v} \Delta v$ of the mean velocity of the beam by its dispersion. The thermalization time between transverse and longitudinal degrees of freedom is then drastically increased by this large mismatch in dispersion velocities.

Another interesting outcome of the model is the distance over which the collision occurs. To calculate the duration $\tau(\xi)$ of the collision, we determine the time $t^{+}(\xi)$ (resp. $t^{-}(\xi)$ ) at which a given atom of initial velocity $v_{\mathrm{i}}-\xi \Delta v$ (resp. $v_{\mathrm{i}}+\xi \Delta v$ ) collides with the moving magnetic wall. We find:

$$
\tau(\xi)=t^{+}(\xi)-t^{-}(\xi)=\frac{2 \xi d \Delta v}{\left(v_{\mathrm{i}}-v_{\mathrm{m}}\right)^{2}-\xi^{2} \Delta v^{2}}
$$

The distance over which the collision occurs is then $\delta(\xi)=$ $v_{\mathrm{m}} \tau(\xi)$. For the parameters of Fig. 2, $v_{\mathrm{i}}=142 \mathrm{~cm} / \mathrm{s}, v_{\mathrm{m}}=$ $88 \mathrm{~cm} / \mathrm{s}$ and $\Delta v=12 \mathrm{~cm} / \mathrm{s}$, we obtain $\tau \simeq 0.4 \mathrm{~s}$ and $\delta \simeq 0.35 \mathrm{~m}$, with $\xi=1.45$ corresponding to $85 \%$ percent of the atoms reflected. This value is in good agreement with the duration over which the mean velocity changes as illustrated in Fig. 3c.

\section{Generation of a continuous beam}

In this section we first summarize the methods that have been demonstrated so far to produce a continuous and magnetically guided atomic beam. We conclude with our preliminary results towards this goal using a moving magnetic mirror, and the improvements that this technique allows.

The realization of a magnetically guided beam has been first achieved by continuously injecting atoms into a magnetic guide using the moving molasses technique 15 , 16. An improvement on the flux by nearly two orders of magnitude $\left(7 \times 10^{9}\right.$ atoms $\left./ \mathrm{s}\right)$ with respect to this latter scheme was obtained by feeding the magnetic guide periodically at a high repetition rate [17. In this scheme, a cold packet of atoms is loaded in an elongated magnetooptical trap $(\mathrm{MOT})$ on a short time $\tau_{\text {feed }} \sim 100 \mathrm{~ms}$. It is then launched into the magnetic guide and after a time delay $\tau_{\text {delay }} \sim 100-200 \mathrm{~ms}$ another packet is prepared. This delay ensures that the light scattered from the MOT during the preparation of the new packet does not generate too many losses on the previous one. The guide is consequently fed at a rate of $1 /\left(\tau_{\text {feed }}+\tau_{\text {delay }}\right) \sim 4 \pm 1$ packets per second. The successive atomic packets then spread according to their dispersion of longitudinal velocities, and eventually overlap. The propagation time required to reach degeneracy through evaporative cooling is then dictated by the kinetics of evaporation in this context 18].

Actually, the delay time $\tau_{\text {delay }}$ is set by the injection velocity. A longer propagation time in the guide requires a priori an injection at lower velocity, which can be done only at the expense of an increase of $\tau_{\text {delay }}$, and therefore of a reduction of the flux. To overcome this limitation, two strategies have been proposed in [14] and experimentally demonstrated in [7]. They consist in slowing down the beam, either by increasing the strength of the transverse 
confinement with a tapered section, or by implementing an upward slope in the guide.

By slowing down of packets of atoms injected into a magnetic guide through their reflection on a moving magnetic mirror, one can use a relatively high injection velocity $v_{\mathrm{i}}$, which permits in turn to decrease $\tau_{\text {delay }}$ when the sequence is repeated. Consequently the flux coupled to the magnetic guide can be increased.

To demonstrate this new method to produce a continuous beam with a very low mean velocity, we inject packets of atoms periodically according to the motion of the mirror on the conveyor belt. We show in Fig. 4 the absorption signal of the probe located in $z_{P}=1.75 \mathrm{~m}$ for successive packets launched into the magnetic guide at a velocity of $v_{\mathrm{i}}=120 \mathrm{~cm} / \mathrm{s}$, in the absence of the moving mirror, and with a mirror set at a velocity $v_{\mathrm{m}}=85 \mathrm{~cm} / \mathrm{s}$. The final velocity of the packet after it has undergone the collision is then $50 \mathrm{~cm} / \mathrm{s}$. With a probe located $50 \mathrm{~cm}$ downstream the first probe we clearly see the onset of overlapping between the successive slowed down packets.

Equation (3) shows that the distance over which the collision occurs can be considerably reduced by a proper choice of the initial velocity of the packets and of the magnetic mirror. For instance, with $v_{\mathrm{i}}=240 \mathrm{~cm} / \mathrm{s}$ and $v_{\mathrm{m}}=140 \mathrm{~cm} / \mathrm{s}$, the collision occurs over a distance of the order of $10 \mathrm{~cm}$. The price to pay is in term of the barrier height that needs to be larger than the kinetic energy of all the particles of the packet for the mirror to be efficient for all atoms. With the latter parameters, $B_{\max }>m\left(v_{\mathrm{i}}-v_{\mathrm{m}}+4 \Delta v\right)^{2} /(2 \mu) \sim 340$ Gauss. For a given final velocity, the higher the injection velocity, the more local the collision. In addition, the duty cycle in the preparation of the packet can be significantly increased, and as a direct consequence the flux also.

In conclusion, we have demonstrated the slowing down of large packets of atoms propagating in a magnetic guide by reflection on a moving magnetic mirror. We have also drawn the perspective of this technique for the generation of a continuous, intense and very slow beam of guided atoms.

We thank Jean Dalibard for a careful reading of the manuscript. We acknowledge financial support from the Délégation Générale pour l'Armement (DGA). Our team belongs to the Institut Francilien de Recherche sur les Atomes Froids (IFRAF). Z. W. acknowledges support from the European Marie Curie Grant MIF1-CT-2004-509423, and G. R. support from the DGA.

\section{References}

1. P. R. Berman, Atom interferometry, (Academic Press, London, 1997).

2. A. Steyerl, H. Nagel, F.-X. Schreiber, K.-A. Steinhauser, R. Ghler, W. Glser, P. Ageron, J. M. Astruc, W. Drexel, G. Gervais, W. Mampe, Phys. Lett. A 116, 347 (1986).

3. S. A. Werner and A. G. Klein, in Neutron Scattering, edited by D. L. Price and K. Skjold, Methods of Experimental Physics Vol. 23 Pt. A (Academic, New York, 1986).

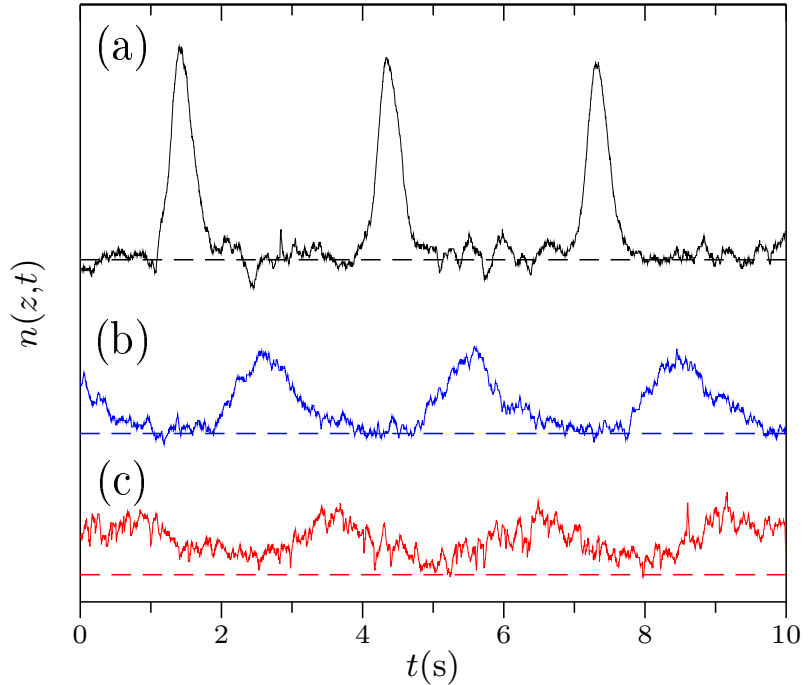

Fig. 4. Multiple injection and slowing of packets of atoms in the magnetic guide. Packets with $v_{\mathrm{i}}=120 \mathrm{~cm} / \mathrm{s}$ probed after a 1.75 meter propagation in the magnetic guide : (a) in absence of the moving mirror, (b) with a mirror set at a velocity $v_{\mathrm{m}}=85$ $\mathrm{cm} / \mathrm{s}$ which allows to reduce the velocity down to $50 \mathrm{~cm} / \mathrm{s}$. (c) Same conditions as (b) but with a probe located at $z_{P}=2.25$ $\mathrm{m}$. Curves have been shifted for clarity reasons, the dashed lines correspond to a vanishing atomic density for each set of data.

4. H. Rauch and S. A. Werner. Neutron interferometry. Number 12 in Oxford Series on Neutron Scattering in Condensed Matter. Oxford University Press, 2000.

5. A. Libson, M. Riedel, G. Bronshtein, E. Narevicius, U. Even, M. G. Raizen, New Journal of Physics B 8, 77 (2006).

6. E. Mandonnet, A. Minguzzi, R. Dum, I. Carusotto, Y. Castin and J. Dalibard, Eur. Phys J. D 10, 9 (2000).

7. T. Lahaye, Z. Wang, G. Reinaudi, S. P. Rath, J. Dalibard, and D. Guéry-Odelin, Phys. Rev. A 72, 033411 (2005).

8. I. G. Hughes, P. A. Barton, T. M. Roach, M. G. Boshier and E. A. Hinds, J. Phys. B: At. Mol. Opt. Phys. 30647 (1997).

9. P. Rosenbusch, B.V. Hall, I.G. Hughes, C.V. Saba, E.A. Hinds, Appl. Phys. B, 70, 709 (2000).

10. A. I. Sidorov, R. J. McLean, W. J. Rowlands, D. C. Lau, J. E. Murphy, M. Walkiewicz, G. I. Opat and P. Hannaford, Quantum Semiclass. Opt. 8, 713 (1996).

11. K. S. Johnson, M. Drndić, J. H. Thywissen, G. Zabow, R. M. Westervelt, and M. Prentiss, Phys. Rev. Lett. 81, 1137 (1998); M. Drndić, G. Zabow, C. S. Lee, J. H. Thywissen, K. S. Johnson, M. Prentiss, R. M. Westervelt, P. D. Featonby, V. Savalli, L. Cognet, K. Helmerson, N. Westbrook, C. I. Westbrook, W. D. Phillips, and A. Aspect, Phys. Rev. A 60, 4012 (1999).

12. A. Steane, P. Szriftgiser, P. Desbiolles, and J. Dalibard, Phys. Rev. Lett. 74, 4972 (1995); M. Arndt, P. Szriftgiser, and J. Dalibard, A. M. Steane, Phys. Rev. A 53, 3369 (1996).

13. H. Goldstein, Classical Mechanics, 2nd edition, Addison Wesley, 1980.

14. T. Lahaye, P. Cren, C. Roos, and D. Guéry-Odelin, Commun. Nonlin. Sci. Numer. Simul. 8, 315 (2003). 
15. P. Cren, C. F. Roos, A. Aclan, J. Dalibard and D. GuéryOdelin, Eur. Phys J. D 20, 107 (2002).

16. Spencer E. Olson, Rahul R. Mhaskar, and Georg Raithel, Phys. Rev. A 73, 033622 (2006).

17. T. Lahaye, J. M. Vogels, K. J. Günter, Z. Wang, J. Dalibard, and D. Guéry-Odelin, Phys. Rev. Lett. 93, 093003 (2004).

18. T. Lahaye and D. Guéry-Odelin, Phys. Rev. A 73, 063622 (2006). 